\title{
Identification of the differentially expressed genes associated with familial combined hyperlipidemia using bioinformatics analysis
}

\author{
XIAOLI LUO, CHANGQING YU, CHUNJIANG FU, WEIBIN SHI, \\ XUKAI WANG, CHUNYU ZENG and HONGYONG WANG \\ Department of Cardiology, Daping Hospital, The Third Military Medical University, \\ Chongqing Institute of Cardiology, Chongqing 400042, P.R. China
}

Received February 12, 2014; Accepted November 18, 2014

DOI: $10.3892 / \mathrm{mmr} .2015 .3263$

\begin{abstract}
The aim of the present study was to screen the differentially expressed genes (DEGs) associated with familial combined hyperlipidemia (FCHL) and examine the changing patterns. The transcription profile of GSE18965 was obtained from the NCBI Gene Expression Omnibus database, including 12 FCHL samples and 12 control specimens. The DEGs were identified using a linear models for microarray data package in the $\mathrm{R}$ programming language. Gene Ontology (GO) function and Kyoto Encyclopedia of Genes and Genomes (KEGG) pathway enrichment analysis was also performed. Protein-protein interaction (PPI) networks of the DEGs were constructed using the EnrichNet online tool. In addition, cluster analysis of the genes in networks was performed using ClusterONE. A total of 879 DEGs were screened, including 394 upregulated and 485 downregulated genes. Enrichment analysis identified four important KEGG pathways associated with FCHL: One carbon pool by folate, $\alpha$-linolenic acid metabolism, asthma and the glycosphingolipid biosynthesis-globo series. GO annotation identified 12 enriched biological processes, including one associated with hematopoiesis and four associated with bone cell differentiation. This identification was in accordance with clinical data and experiments into hyperlipidemia and bone lesions. Based on PPI networks, these DEGs had a close association with immune responses, hormone responses and cytokine-cytokine receptors. In conclusion, these DEGs may be used as specific therapeutic molecular targets in the treatment of FCHL. The present findings may provide the basis for understanding the
\end{abstract}

Correspondence to: Dr Hongyong Wang, Department of Cardiology, Daping Hospital, The Third Military Medical University, Chongqing Institute of Cardiology, 10 Yangtze River Bypass Road, Daping, Yuzhong, Chongqing 400042, P.R. China E-mail: whysir@aliyun.com

Key words: familial combined hyperlipidemia, differentially expressed genes, function enrichment analysis, protein-protein interaction network pathogenesis of FCHL in future studies. However, further experiments are required to confirm these results.

\section{Introduction}

Familial combined hyperlipidemia (FCHL), the most common genetic form of hyperlipidemia, is characterized by significant familial clustering and premature coronary heart disease (1). FCHL is a common inherited disorder of lipid metabolism with a prevalence of $0.5-2.0 \%$, accounting for $10 \%$ of the cases of premature coronary heart disease worldwide (2). Therefore, the research and treatment of FCHL has significance for human health. Multiple hyperlipemic phenotypes have been characterized in the same individual and in the same family, which can be detected by elevated very-low-density lipoproteins (VLDL) and low-density lipoproteins (LDL) or apolipoprotein B (apoB) $(3,4)$.

To date, studies have focused on the molecular mechanisms of FCHL development in order to reveal biomarkers for clinical treatment. The FCHL locus has been mapped to human chromosome 1q21-q23. This region includes retinoid $X$ receptor $\gamma$ (RXRG), a nuclear factor member of the RXR superfamily, which is critical in lipid homeostasis (1). Sentinelli et al (1) have identified five polymorphisms in the RXRG gene (rs1128977, rs2651860, rs2134095, rs283696 and rs10918169). Hsieh et al (5) suggested that one single nucleotide polymorphism (SNP) in the RXRG gene, (rs3818569 now merged into rs1128977) has a positive correlation with the development of diabetic retinopathy. The rs2651860 SNP was significantly associated with increased levels of LDL-cholesterol and of apoB in T-allele carriers (1). A total of three SNPs in RXR $\hat{\mathrm{I}}^{3}$ exhibited a significant association with HIV lipodystrophy (6).

In previous years, multiple candidate genes have been identified as associated with the FCHL phenotype. The upstream transcription factor 1 (USF1) is a transcription factor, which regulates the expression of a number of genes involved in glucose and lipid metabolism, and provides an adequate candidate for FCHL (7). Preliminary functional data suggested that the USF1 risk haplotype may affect the expression profiles in fat biopsy samples from individuals with FCHL (8). The lipoprotein lipase (LPL) gene is also a noteworthy candidate for FCHL. The decreased activity of LPL in subjects with FCHL has been identified and positive associations have 
been reported between FCHL and genetic variants in the LPL promoter and exon (9). In brief, these candidate gene studies may provide a theoretical foundation for FCHL treatment.

In the present study, the aim was to analyze the FCHL samples and control samples with a series of biological information technology services, with the purpose of revealing the mechanism underlying the development of FCHL. Gene-set enrichment analysis was performed and a protein-protein interaction (PPI) network was constructed. Functional genes and signaling pathways in FCHL were used to establish a theoretical foundation for future research. Present findings may provide a basis for understanding the pathogenesis of FCHL in the future.

\section{Materials and methods}

Data sources. The transcription profile of GSE1010 was obtained from the NCBI Gene Expression Omnibus (GEO) database (http:/www.ncbi.nlm.nih.gov/geo/), which is based on the Affymetrix Human Genome U133A Array GPL96 (Affymetrix, Santa Clara, CA, USA). There were a total of 24 RNA specimens (lymphoblastic cells), including 12 FCHL samples and 12 control specimens.

Screening of differentially expressed genes (DEGs). The linear models for microarray data package (10) in the R programming language was used to identify DEGs. The original expression datasets were normalized using the normalize within arrays method and normalize between arrays method (11). Following normalization, the expression value was used to construct a linear model in order to identify the DEGs (12). $\mathrm{P}<0.05$ was set as the cut-off criteria.

Kyoto Encyclopedia of Genes and Genomes (KEGG) pathway analysis based on the PPI network. The KEGG pathway enrichment analysis of DEGs was performed using EnrichNet (http://www.enrichnet.org/) (13). EnrichNet is an analysis approach based on the PPI networks. EnrichNet calculates the overlap between the known KEGG pathways and constructed PPI networks, in order to acquire the PPI enriched KEGG pathway. In the present study, PPI networks of DGEs were constructed via the Search tool for the retrieval of interacting genes/proteins (STRING; http://www.string-db. org/) (14) database and the similarity between the PPI networks and the KEGG pathways were calculated via EnrichNet. The similarity was presented as an XD-score. The higher the XD-score value, the higher the similarity is, indicating an increased possibility of a KEGG pathway enriched with DEGs. In order to notarize the criteria of the XD-score, the classical overlap-based Fisher test was used to calculate the significance score (q-value) via EnrichNet and linear regression analysis between the q-value and XD-score was performed. An XD-score lower than the threshold value of 0.79 , corresponding to a q-value of 0.05 was considered to indicate significance.

Construction of the PPI network combined with the KEGG pathway. For the enriched KEGG pathways, the integrated PPI combined with the KEGG pathways was constructed via EnrichNet, based on the PPI network of the DEGs. Briefly, the PPI network was presented via Cytoscape (http://cytoscape. org/) (15) and then integrated with the PPI associated with
Table I. Identification of differentially expressed genes associated with familial combined hyperlipidemia with $\mathrm{P}<0.05$.

\begin{tabular}{lcc}
\hline Gene symbol & P-value & Log $_{2} \mathrm{FC}$ \\
\hline SSX4B///SSX4 & $5.06 \mathrm{E}-05$ & -0.818 \\
TAS2R10 & $6.16 \mathrm{E}-05$ & -1.004 \\
FBXO2 & $6.61 \mathrm{E}-05$ & -0.357 \\
ASMT & $1.16 \mathrm{E}-04$ & -0.578 \\
BMP7 & $1.50 \mathrm{E}-04$ & -0.394 \\
PCSK1 & $1.55 \mathrm{E}-04$ & -0.750 \\
PSD3 & $3.55 \mathrm{E}-04$ & -0.392 \\
GTF2H3 & $5.54 \mathrm{E}-04$ & -0.525 \\
BTNL8 & $5.67 \mathrm{E}-04$ & -0.619 \\
VIL1 & $7.08 \mathrm{E}-04$ & -0.592 \\
RBM12B & $4.92 \mathrm{E}-05$ & 0.556 \\
TFF1 & $1.84 \mathrm{E}-04$ & 0.470 \\
KANSL1L & $1.88 \mathrm{E}-04$ & 0.651 \\
SOX11 & $2.13 \mathrm{E}-04$ & 0.590 \\
UMPS & $5.59 \mathrm{E}-04$ & 0.569 \\
RAP1GAP & $6.81 \mathrm{E}-04$ & 0.380 \\
C2Orf83 & $7.06 \mathrm{E}-04$ & 0.418 \\
SDC4 & $7.14 \mathrm{E}-04$ & 0.181 \\
MAST2 & $9.48 \mathrm{E}-04$ & 0.407 \\
AFF2 & $1.06 \mathrm{E}-03$ & 0.223 \\
& &
\end{tabular}

Predominant upregulated and downregulated differentially expressed genes are listed.

the significant KEGG pathways, exhibiting the distribution and mutual connection association of the significant KEGG pathways in the integrated PPI network.

Protein complexes predicted via ClusterONE. ClusterONE is a graph-clustering algorithm, which is used for forecasting the potential protein complexes in the weighted PPI (16). The weight of the PPI was set as the score provided by STRING.

Subsequently, the predicted protein complexes were verified. A protein complex enriched into a KEGG pathway, a protein domain or a cellular component was identified as a potential protein with function. The protein domain and cellular component were analyzed via the database for annotation, visualization and integrated discovery (17) based on the InterPro (http://www. ebi.ac.uk/interpro/) (18) database and the gene ontology (GO) cellular component to conduct enrichment analysis.

GO enrichment analysis of the PPI network. GO gene annotation of the PPI network was performed via EnrichNet. GO terms were classified into biological process (BP) and molecular function (MF). The Pearson correlation coefficient was 0.8 and the threshold value for the XD-score was 1.68 .

\section{Results}

Identification of DEGs. To identify the specific DEGs between human FCHL tissues and healthy controls, the publicly available microarray dataset, GSE1010 was obtained from 
Table II. Main KEGG pathways of differentially expressed genes.

\begin{tabular}{lccr}
\hline KEGG pathway & XD-score & Fisher q-value & Gene list $^{\mathrm{b}}$ \\
\hline hsa00670 & 1.064 & 0.331 & MTHFS, MTHFR, SHMT1 \\
hsa00592 & 0.976 & 0.331 & PLA2G2E, PLA2G3, PLA2G12A \\
hsa05310 & 0.928 & 0.301 & FCER1A, HLA-DPA1, \\
hsa00603 & $0.762^{\mathrm{a}}$ & 0.460 & HLA-DRB1, IL3, CD40 \\
& & ST8SIA1, B3GALNT1
\end{tabular}

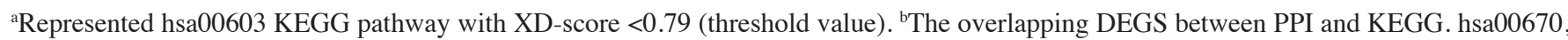
one carbon pool by folate; hsa00592, $\alpha$-linolenic acid metabolism; hsa05310, asthma; hsa00603, glycosphingolipid biosynthesis-globo series; PPI, protein-protein interaction; KEGG, Kyoto Encyclopedia of Genes and Genomes.

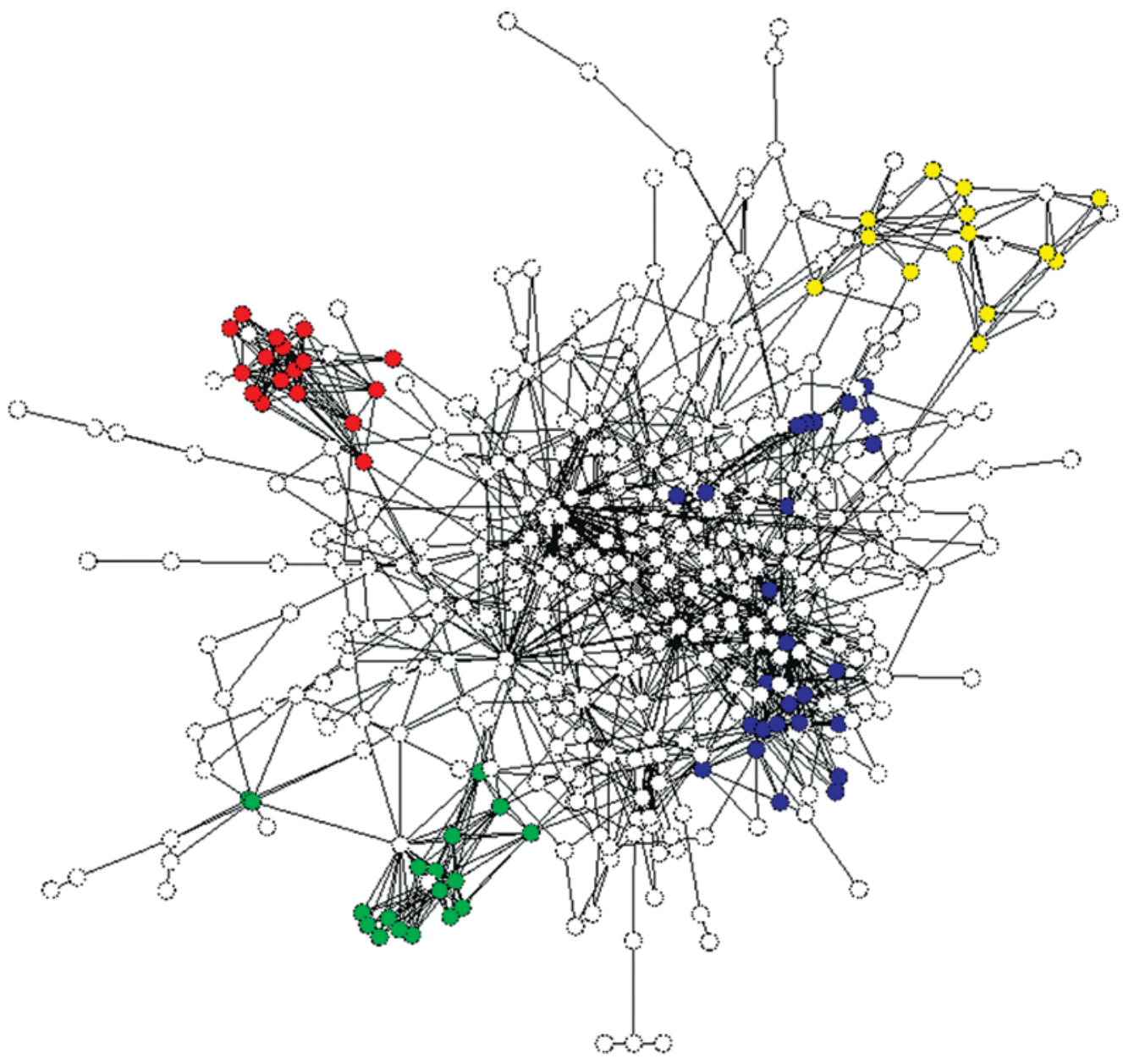

Figure 1. PPI network construction of DEGs combined with KEGG pathway analysis. The colored nodes represent DEGs involved in the four main KEGG pathways. Red indicates hsa00670: One carbon pool by folate, green indicates hsa00592: $\alpha$-linolenic acid metabolism, blue indicates hsa05310: asthma and yellow indicates hsa00603: Glycosphingolipid biosynthesis-globo series. DEG, differentially expressed genes; PPI, protein-protein interaction; KEGG, Kyoto Encyclopedia of Genes and Genomes.

the GEO database. The gene expression profiling data were preprocessed using the Affy package and were normalized by the median method. At $\mathrm{P}<0.05$, a total of 879 DEGs were identified, including 394 upregulated and 485 downregulated genes, of which the 10 most predominant upregulated and downregulated genes are listed in Table I.

Construction of the PPI network of DEGs. PPI networks of the DEGs were constructed via STRING. Subsequently, the KEGG pathway enrichment analysis of DEGs in the PPI networks was performed using EnrichNet. The PPI network included 431 nodes and 1,124 associations (Fig. 1). The predominant four KEGG pathways were identified(Table II), including one carbon pool by folate (hsa00670; XD-score $=1.064), \alpha$-linolenic acid metabolism (hsa00592; XD-score=0.976), asthma (hsa05310; $\mathrm{XD}$-score $=0.928$ ), with an $\mathrm{XD}$-score $\geq 0.79$ and glycosphingolipid biosynthesis-globo series (hsa00603, XD-score=0.762). The enrichment of the glycosphingolipid biosynthesis pathway 
A

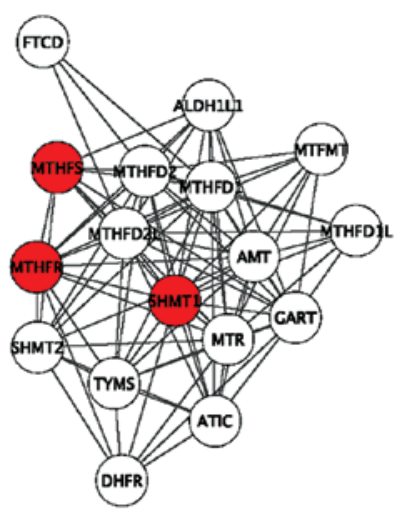

C

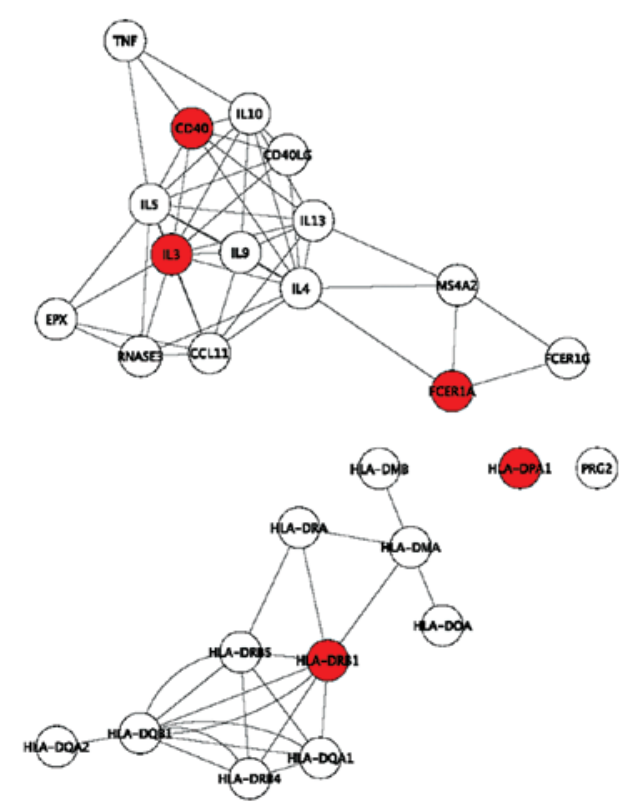

B

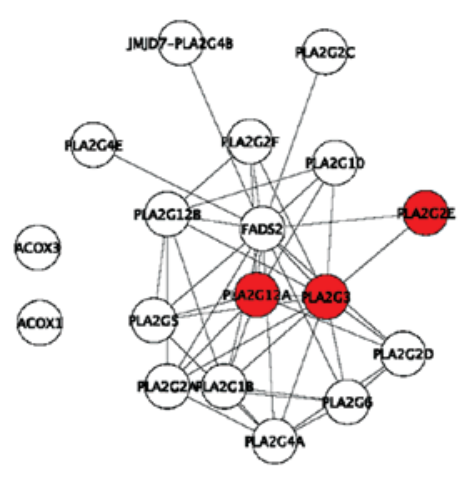

D

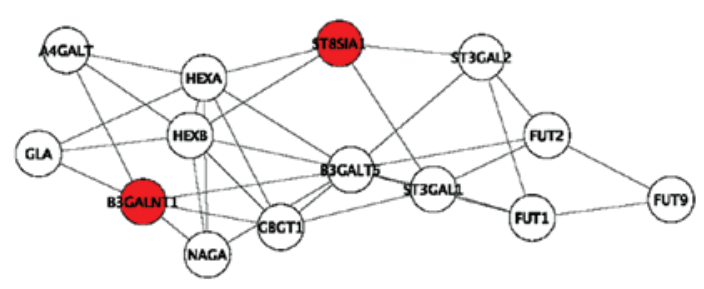

Figure 2. PPI network of DEGs involved in the four main KEGG pathways. (A) Hsa00670, One carbon pool by folate; (B) hsa00592, $\alpha$-linolenic acid metabolism; (C) hsa05310, asthma; and (D) Hsa00603, glycosphingolipid biosynthesis-globo series. Red nodes indicate the overlapping DEGs between the KEGG pathway and the PPI constructed by the DEGs. DEG, differentially expressed genes; PPI, protein-protein interaction; KEGG, Kyoto Encyclopedia of Genes and Genomes.

may be undervalued by EnrichNet. The DEGs involved in the four main KEGG pathways are in color within the diagram of the PPI network (Fig. 1).

PPI network of DEGs involved in the KEGG pathway. The PPI network of DEGs involved in the main four KEGG pathways is shown in Fig. 2, in which the red nodes represent the DEGs. A number of DEGs were located in the center of the PPI network. SHMT1 had a high betweenness value (0.0382) in hsa00670 and it was ranked fifth in all nodes. (Fig. 2A). Additionally, the KEGG pathway of hsa00670 is shown in Fig. 3. SHMT1 encodes serine hydroxymethyltransferase 1, represented as EC 2.1.2.1 (Fig. 3) and is important in this pathway as it catalyzes the hydrolysis of tetrahydrofolate (THF) into 5, 10-methylene-THF. DEGs in other pathways with high betweenness included PLA2G and PLA2G12A in hsa00592 (Fig. 2B), HLA-DRB1 in hsa05310 (Fig. 2C) and B3GALNT1 in hsa00603 (Fig. 2D).

ClusterONE prediction of protein complexes and its validation. At $\mathrm{P}<0.01$, a total of 10 protein complexes were predicted via ClusterONE (Table III). The genes of Complexes 1-5 exhibited overlap with the four main KEGG pathways, indicating that these genes were differentially enriched in the four KEGG pathways. At the same time, the protein domain classification of DEGs involved in Complexes 1-5 demonstrated that these DEGs were from the same enzyme or signaling molecules and were regulatory in the corresponding KEGG pathway. In addition, the cellular components of these DEGs was consistent.

Complex 6 revealed no KEGG pathway enrichment, but these DEGs were members of the $\mathrm{G}$ protein-coupled receptor (GPCR) family, of which $75 \%$ were localized in the cell membrane. Approximately $43 \%$ of the DEGs of Complex 7 were localized in the synaptic vesicles and the DEGs of Complex 10 were enriched in protein domains without the determined localization. It was difficult to determine whether Complex 7 and 10 may have biological functions. In addition, the function of Complexes 8 and 9 was not verified, which may be due to an error with ClusterONE.

GO gene annotation of DEGs. GO gene annotation of DEGs revealed 12 BP GO terms (Table IV), including four GO 
Table III. Predicted protein complexes via ClusterONE.

\begin{tabular}{|c|c|c|c|c|c|c|}
\hline Rank & Protein domains & $\begin{array}{l}\text { Relevant } \\
\text { pathway }\end{array}$ & $\begin{array}{l}\text { Cellular } \\
\text { component }^{\mathrm{a}}\end{array}$ & $\begin{array}{l}\text { Gene } \\
\text { count }\end{array}$ & Quality $^{\mathrm{b}}$ & P-value \\
\hline 1 & Phospholipase A2 & hsa00592 & Extracellular region $(63 \%)$ & 20 & 0.878 & $4.84 \mathrm{E}-08$ \\
\hline 2 & $\begin{array}{l}\text { THF dehydrogenase; } \\
\text { formyl transferase; SHMT; }\end{array}$ & hsa00670 & Mitochondrion (44\%) & 18 & 0.891 & $1.79 \mathrm{E}-07$ \\
\hline 3 & $\begin{array}{l}\text { Sialyltransferase; } \\
\text { GTF, family } 31 \text {; } \\
\text { GTF, family } 11 ; \\
\text { GH, family } 20\end{array}$ & hsa00603 & Golgi apparatus (76\%) & 17 & 0.756 & $2.77 \mathrm{E}-06$ \\
\hline 4 & $\begin{array}{l}\text { Four-helical cytokine, core; } \\
\text { IL-4; IL-17; TNF 2; } \\
\text { Peroxidases heam-ligand } \\
\text { binding site; Toll-IL R } \\
\text { MHC class I, } \alpha \text { chain, } \\
\alpha 1 \text { and } \alpha 2\end{array}$ & hsa05310 & Extracellular space (52\%) & 27 & 0.598 & $3.72 \mathrm{E}-06$ \\
\hline 5 & Immunoglobulin C-Type & hsa05310 & $\begin{array}{l}\text { MHC class II protein } \\
\text { complex }(100 \%)\end{array}$ & 9 & 0.793 & $5.98 \mathrm{E}-04$ \\
\hline 6 & $\begin{array}{l}\text { GPCR, rhodopsin-like } \\
\text { superfamily }\end{array}$ & NA & $\begin{array}{l}\text { Integral to plasma } \\
\text { membrane (75\%) }\end{array}$ & 12 & 0.578 & $7.86 \mathrm{E}-04$ \\
\hline 7 & NA & NA & Synaptic vesicle (43\%) & 8 & 0.701 & 0.001 \\
\hline 8 & NA & NA & NA & 6 & 0.645 & 0.005 \\
\hline 9 & NA & NA & NA & 6 & 0.625 & 0.007 \\
\hline 10 & $\begin{array}{l}\text { 3'5'-cyclic nucleotide PDE; } \\
\text { adenylyl cyclase class-3/4/ } \\
\text { guanylyl cyclase, conserved site }\end{array}$ & NA & NA & 7 & 0.711 & 0.007 \\
\hline
\end{tabular}

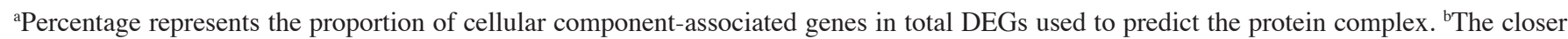
the value is to 1 , the higher the possibility of a predicted compound. THF, tetrahydrofolate; SHMT, serine hydroxymethyltransferase; GTF, glycosyl transferase; GH, Glycoside hydrolase; IL, interleukin; TNF, tumor necrosis factor; R, receptor; PDE, phosphodiesterase.

Table IV. GO gene annotation of differentially expressed genes via EnrichNet.

\begin{tabular}{lccr}
\hline GO (biological process) & XD-score & $\begin{array}{c}\text { Fisher } \\
\text { q-value }\end{array}$ & $\begin{array}{r}\text { Gene } \\
\text { count }\end{array}$ \\
\hline GO:0048762 (mesenchymal cell differentiation) & 4.033 & 0.018 & 5 \\
GO:0032331 (negative regulation of chondrocyte differentiation) & 2.303 & 0.162 & 4 \\
GO:0045885 (positive regulation of survival gene product expression) & 2.303 & 0.162 & 4 \\
GO:0060445 (branching involved in salivary gland morphogenesis) & 2.303 & 0.162 & 4 \\
GO:0007567 (parturition) & 2.233 & 0.280 & 3 \\
GO:0090009 (primitive streak formation) & 2.233 & 0.280 & 3 \\
GO:0060740 (prostate gland epithelium morphogenesis) & 2.233 & 0.280 & 3 \\
GO:0045667 (regulation of osteoblast differentiation) & 2.105 & 0.194 & 3 \\
GO:0030278 (regulation of ossification) & 1.988 & 0.317 & 3 \\
GO:0010039 (response to iron ion) & 1.933 & 0.208 & 3 \\
GO:0071425 (hemopoietic stem cell proliferation) & 1.783 & 0.332 & 3 \\
GO:0018298 (protein-chromophore linkage) & 1.783 & 0.332 & 3 \\
\hline
\end{tabular}

terms associated with bone cell proliferation (GO: 0048762 mesenchymal cell differentiation, GO: 0032331 negative regulation of chondrocyte differentiation, GO: 0045667 regulation of osteoblast differentiation and GO: 0030278 regulation of ossification) and one GO term associated with blood (GO: 0071425 hemopoietic stem cell proliferation). The enriched 


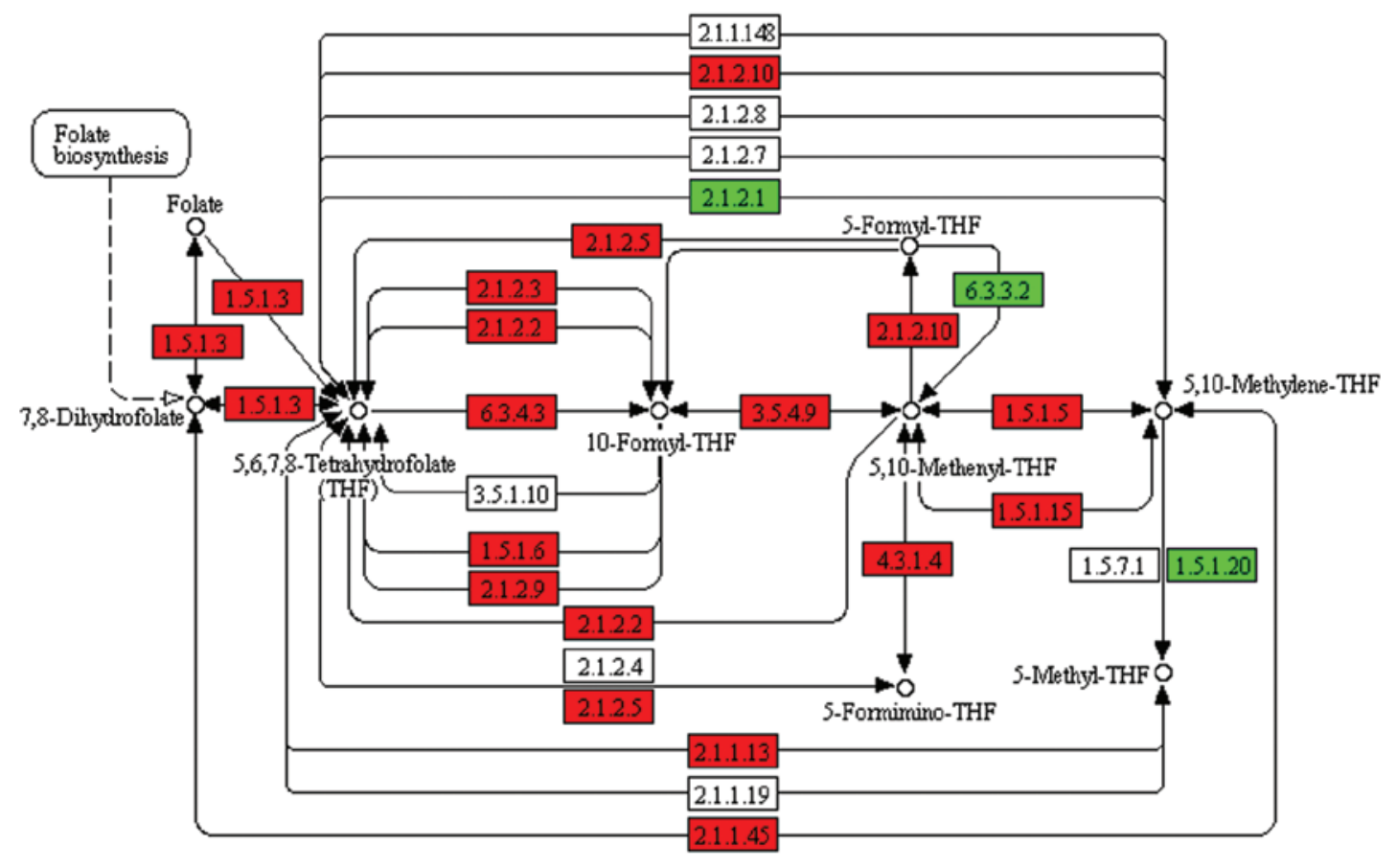

$006709 / 13 / 11$

(c) Kanehisa Laboratories

Figure 3. KEGG pathway of hsa00670: one carbon pool by folate. Red indicates the genes involved in the hsa00670 via EnrichNet. Green indicates the overlapping DEGs between the KEGG pathway and the PPI constructed by the DEGs. DEGs, differentially expressed genes; PPI, protein-protein interaction; KEGG, Kyoto Encyclopedia of Genes and Genomes.

GO terms associated with bone cells were consistent with the phenomenon that hyperlipidemia causes bone lesions in experimental and clinical settings.

\section{Discussion}

The present study used the EnrichNet online database to analyze RNA samples from patients with FCHL. Initially, the PPI network was constructed for DEGs, subsequently PPI and KEGG pathways were compared in the database (or GO gene annotation) and the KEGG pathway enrichment in DEGs (or GO terms) were identified. KEGG pathway analysis identified four important KEGG pathways, including one carbon pool by folate (hsa00670), $\alpha$-linolenic acid metabolism (hsa00592), asthma (hsa05310) and the glycosphingolipid biosynthesis-globo series (hsa00603). The one carbon pool by folate pathway consists of the folic acid synthesis of folate THF biosynthesis and the $\mathrm{C} 1$-unit conversion process. THF, a carrier of the one-carbon group, acts as a coenzyme DNA-synthesis of nucleic acid and a lack of THF can lead to anemia (19). The $\alpha$-linolenic acid metabolism KEGG pathway is associated with fatty acid $\alpha$-linolenic acid metabolism. $\alpha$-linolenic acid can reduce cholesterol levels in the blood (20) and alleviate the effect of hyperlipidemia $(21,22)$. The asthma pathway enriched by DEGs of patients with hyperlipidemia may be associated with evidence suggesting that hyperlipidemia may cause asthma-associated complications (23). Glycosphingolipid synthesized via the glycosphingolipid biosynthesis-globo series pathway may accumulate in the artery wall and precipitate, which is an established feature of atherosclerosis (24). When this pathway is inhibited, the cholesterol content in the blood is reduced and the degree of atherosclerosis is alleviated (25). The important effect of glycosphingolipid on hyperlipidemia has been discussed previously (26).

Parhami (27) summarized the effects of hyperlipemia on osteoporosis as several patients with atherosclerosis also suffer from osteoporosis. This review suggests that hyperlipemia is the cause of osteoporosis. Further studies have also discussed the association between pathological changes of bone tissue and hyperlipidemia $(28,29)$. For example, although Complex 6 exhibited no KEGG pathway enrichment, these DEGs were identified as members of the GPCR family, of which $75 \%$ were localized in the cell membrane. GPCRs have provided novel opportunities for structure-based drug design strategies targeting this protein family (30).

GO function analysis identified 12 enriched BP terms, of which one term was associated with hematopoiesis and four terms were associated with bone cell differentiation. This finding was in accordance with hyperlipidemia and bone lesions in clinical and experimental settings. To date, clinical trials for the treatment of ischemic heart disease and heart failure using bone marrow cells have rapidly increased (31). Baldán et al (32) have demonstrated that diet-induced atherosclerosis is impaired when atherosclerotic-susceptible mice are transplanted with ATP-binding cassette sub-family G member $1(\text { Abcg } 1)^{-/-}$bone marrow. The demonstration that 
$\mathrm{Abcg}^{-/-}$macrophages undergo accelerated apoptosis provides a mechanism to explain the decrease in atherosclerotic lesions. Drechsler et al (33) provided evidence that hypercholesterolemia-induced neutrophilia is multifactorial and that neutrophils infiltrate arteries primarily during early stages of atherosclerosis, which also supports the present results.

In conclusion, the current study identified 897 DEGs and analyzed their functions. Additionally, bioinformatics methods were used to analyze the overlapping DEGs with known genes of the KEGG pathways. Subsequently, the enriched GO terms of DEGs were analyzed. The present study may provide a basis for improved understanding of FCHL. However, experimental studies are required to confirm these findings.

\section{References}

1. Sentinelli F, Minicocci I, Montali A, et al: Association of RXR-Gamma gene variants with Familial combined hyperlipidemia: Genotype and haplotype analysis. J Lipids 2013: 517943, 2013

2. Wojciechowski AP, Farrall M, Cullen P, et al: Familial combined hyperlipidaemia linked to the apolipoprotein AI-CIII-AIV gene cluster on chromosome 11q23q-q24. Nature 349: 161-164, 1991.

3. Ayyobi AF, McGladdery SH, McNeely MJ, Austin MA Motulsky AG and Brunzell JD: Small, dense LDL and elevated apolipoprotein B are the common characteristics for the three major lipid phenotypes of familial combined hyperlipidemia. Arterioscler Thromb Vasc Biol 23: 1289-1294, 2003.

4. Sniderman A and Ribalta J: How should FCHL be defined and how should we think about its metabolic bases? Nutr Metab Cardiovasc Dis 11: 259-273, 2001

5. Hsieh C, Pei D, Hung Y and Hsiao F: Association between retinoid-X receptor-gamma genetic polymorphisms and diabetic retinopathy. Genet Mol Res 10: 3545-3551, 2011.

6. Pushpakom SP, Owen A, Back DJ and Pirmohamed M RXR $\gamma$ gene variants are associated with HIV lipodystrophy. Pharmacogenet Genomics 23: 438-441, 2013.

7. Wang Y, Xue F, Liu L and He Z: Pathway analysis detect potential mechanism for familial combined hyperlipidemia. Eur Rev Med Pharmacol Sci 17: 1909-1915, 2013.

8. Pajukanta P, Lilja HE, Sinsheimer JS, et al: Familial combined hyperlipidemia is associated with upstream transcription factor 1 (USF1). Nat Genet 36: 371-376, 2004.

9. Coon H, Myers RH, Borecki IB, et al: Replication of linkage of Familial combined hyperlipidemia to chromosome 1q with additional heterogeneous effect of apolipoprotein AI/C-III/A-IV locus The NHLBI Family Heart Study. Arterioscler Thromb Vasc Biol 20: 2275-2280, 2000.

10. Smyth GK: Limma: Linear Models for Microarray Data. In: Bioinformatics and Computational Biology Solutions Using $\mathrm{R}$ and Bioconductor. Gentleman R, Carey V, Huber W, Irizarry R and Dudoit S (eds). Springer, New York, NY pp397-420, 2005.

11. Ritchie ME, Silver J, Oshlack A, et al: A comparison of background correction methods for two-colour microarrays. Bioinformatics 23: 2700-2707, 2007.

12. Smyth GK: Linear models and empirical bayes methods for assessing differential expression in microarray experiments. Stat Appl Genet Mol Biol 3: 3, 2004

13. Glaab E, Baudot A, Krasnogor N, Schneider R and Valencia A EnrichNet: network-based gene set enrichment analysis. Bioinformatics 28: i451-i457, 2012.
14. Jensen LJ, Kuhn M, Stark M, et al: STRING 8-a global view on proteins and their functional interactions in 630 organisms. Nucleic Acids Res 37: D412-D416, 2009.

15. Shannon P, Markiel A, Ozier O, et al: Cytoscape: a software environment for integrated models of biomolecular interaction networks. Genome Res 13: 2498-2504, 2003.

16. Nepusz T, Yu H and Paccanaro A: Detecting overlapping protein complexes in protein-protein interaction networks. Nat Methods 9: 471-472, 2012.

17. Dennis Jr G, Sherman BT, Hosack DA, et al: DAVID: database for annotation, visualization and integrated discovery. Genome Biol 4: P3, 2003

18. Hunter S, Jones P, Mitchell A, et al: InterPro in 2011: new developments in the family and domain prediction database. Nucleic Acids Res 40: D306-D312, 2012.

19. Locasale JW: Serine, glycine and one-carbon units: cancer metabolism in full circle. Nat Rev Cancer 13: 572-583, 2013.

20. Almario RU, Vonghavaravat V, Wong R and Kasim-Karakas SE Effects of walnut consumption on plasma fatty acids and lipoproteins in combined hyperlipidemia. Am J Clin Nutr 74: 72-79, 2001.

21. Alessandri C, Pignatelli P, Loffredo L, et al: Alpha-linolenic acid-rich wheat germ oil decreases oxidative stress and CD40 ligand in patients with mild hypercholesterolemia. Arterioscler Thromb Vasc Biol 26: 2577-2578, 2006

22. Zhao G, Etherton TD, Martin KR, West SG, Gillies PJ and Kris-Etherton PM: Dietary $\alpha$-linolenic acid reduces inflammatory and lipid cardiovascular risk factors in hypercholesterolemic men and women. J Nutr 134: 2991-2997, 2004.

23. Al-Shawwa B, Al-Huniti N, Titus G and Abu-Hasan M: Hypercholesterolemia is a potential risk factor for asthma. J Asthma 43: 231-233, 2006.

24. Nakou E, Babageorgakas P, Bouchliou I, et al: Statin-induced immunomodulation alters peripheral invariant natural killer T-cell prevalence in hyperlipidemic patients. Cardiovasc Drugs Ther 26: 293-299, 2012

25. Bietrix F, Lombardo E, van Roomen CP, et al: Inhibition of glycosphingolipid synthesis induces a profound reduction of plasma cholesterol and inhibits atherosclerosis development in APOE*3 Leiden and low-density lipoprotein receptor-/- mice. Arterioscler Thromb Vasc Biol 30: 931-937, 2010.

26. Hara A and Taketomi T: Characterization and changes of glycosphingolipids in the aorta of the Watanabe hereditable hyperlipidemic rabbit. J Biochem 109: 904-908, 1991.

27. Parhami F: Possible role of oxidized lipids in osteoporosis: could hyperlipidemia be a risk factor? Prostaglandins, leukotrienes and essential fatty acids 68: 373-378, 2003.

28. Solomon DH, Avorn J, Canning CF and Wang PS: Lipid levels and bone mineral density. Am J Med 118: 1414. 2005.

29. Schmiedl A, Schwille P, Bonucci E, Erben R, Grayczyk A and Sharma V: Nephrocalcinosis and hyperlipidemia in rats fed a cholesterol-and fat-rich diet: association with hyperoxaluria, altered kidney and bone minerals and renal tissue phospholipid-calcium interaction. Urol Res 28: 404-415, 2000.

30. Rosenbaum DM, Cherezov V, Hanson MA, et al: GPCR engineering yields high-resolution structural insights into 32-adrenergic receptor function. Science 318: 1266-1273, 2007.

31. Liao J, Chen X, Li Y, et al: Transfer of bone-marrow-derived mesenchymal stem cells influences vascular remodeling and calcification after balloon injury in hyperlipidemic rats. J Biomed Biotechnol 2012: 165296, 2012

32. Baldán Á, Pei L, Lee R, et al: Impaired development of atherosclerosis in hyperlipidemic Ldlr-/- and ApoE-/- mice transplanted with Abcg1-/- bone marrow. Arterioscler Thromb Vasc Biol 26: 2301-2307, 2006

33. Drechsler M, Megens RT, van Zandvoort M, Weber C and Soehnlein O: Hyperlipidemia-triggered neutrophilia promotes early atherosclerosis. Circulation 122: 1837-1845, 2010. 